\title{
Using Evolved Analogies to Overcome Creative Design Fixation
}

\author{
Steven M. Smith, Julie Linsey and A. Kerne \\ Texas A\&M University, USA
}

\begin{abstract}
Human cognition is critically important in all creative conceptual design. People are susceptible to design fixation, blocks or impasses caused by a variety of unconscious cognitive processes. Insight that resolves fixation can be triggered by accidentally encountered cues, but designers cannot know in advance which environmental triggers are most appropriate. Two domains, patents and life forms, encompass countless well-tested mechanisms for solving environmental problems. A patent database and a compendium of life forms could provide rich sources of analogies that might trigger insight, thereby overcoming design fixation.
\end{abstract}

Keywords: creativity, cognition, fixation, incubation, insight, analogy, biological

\section{Designers Are People}

It may seem obvious, once you see it written down or spoken aloud, that designers are humans. Therefore, every design that is created is conceptualized by a human mind, which is the purview of cognitive psychology. Cognitive psychologists conduct experiments to test theories about cognitive structures (such as "working memory" or "mental models") and cognitive processes (such as "encoding" or "visualization"). The various ways in which cognitive structures and processes collaborate to produce creative ideas is referred to as "creative cognition" (e.g., Finke, Ward \& Smith, 1992; Smith, Ward \& Finke, 1995). Rather than focusing on some singular "creative process," the creative cognition approach portrays creative thinking as a set of skills, operations, and methods for producing creative ideas.

Creative cognition, that is, creative thinking engages many different cognitive mechanisms. Some of the more prominent ones include problem solving, conceptualization, analogical reasoning, inductive inference, conceptual combination, and visualization. These cognitive mechanisms are found in essentially all humans; what is special about creative cognition are not these underlying structures and processes, but rather the ways in which individuals engage them. Different domains of creativity, such as business, musical performance, or science, may have very different ways in which creative contributions are produced and discovered, and even within a single discipline, different individuals may utilize different approaches for thinking creatively. Nonetheless, there are a few universals that appear in virtually all domains and individuals, and scientists have studied these regularities to better understand creative cognition.

\section{Cognitive Fixation}

Cognitive fixation refers to a potentially resolvable block or impediment to reaching the goal of one's mental activity, something that blocks completion of different types of cognitive operations, including many processes and structures involved in memory, problem solving, and creative ideation (e.g., Chrysikou \& Weisberg, 2005; Jansson \& Smith, 1991; Purcell \& Gero, 1996; Smith \& Blankenship, 1989, 1991). The cognitive operations that cause fixation are usually adaptive.

An unconscious cognitive system that reacts to stimuli and situations, enabling automatic responses to long-practiced skills like reading, driving, or recognizing familiar faces. This system provides the means for "offloading" (cognitively) the processing of those frquent responses to the automatic system, rather than using up resources of the conscious explicit system. This offloading allows more resources for the conscious (explicit) system for complex tasks, ones that are not represented by rote responses. The representations that, through one's learned skills, can be offloaded to an unconscious system is adaptive; consequently, the rare inappropriate use of unconsciously-processed knowledge is difficult to detect. A persistent and implicit use of knowledge that is inappropriate and counterproductive is a good definition of fixation. 


\subsection{Implicit Memory}

Implicit memory, an unconscious memory system, remains intact even in amnesic patients who have poor explicit memory, our conscious memory system. After reading a list of words that includes the word ANALOGY, most people, including amnesiacs, find it easy to complete the word fragment $A_{-} L_{-} G$ Y a short time later. Having recently seen the solution word, their implicit memory brings the correct solution ANALOGY immediately to mind, without the need for deliberate attempts to think of a solution. Smith \& Tindell (1997), and later, others (e.g., Lustig \& Hasher, 2001; Leynes, Rass \& Landau, 2008; Kinoshita \& Towgood, 2001), showed that the word fragment $\mathrm{A}_{-} \mathrm{L}_{-} \mathrm{G}_{\mathrm{Y}}$ is particularly difficult to solve, and for the same reason; in this case, implicit memory brings instantly to mind the incorrect answer, ANALOGY, which orthographically resembles the correct answer, ALLERGY. Smith \& Tindell showed that people cannot avoid this type of cognitive fixation even when they are explicitly warned about it.

\subsection{Problem Solving}

Studies of fixation effects in creative problem solving (Kohn \& Smith, 2009; Smith \& Blankenship, 1989, 1991) found that showing subjects inappropriate hints interferes with the ability to solve creative puzzle problems, such as Remote Associates Test problems. In these experiments, participants had to think of solution words that were remotely related to all three test words in each puzzle problem. Participants who read non-solution words that were closely related to test words were significantly less able to think of the appropriate remote associate solution words.

\subsection{Creative Idea Generation}

People who have seen or heard inappropriate "hints" have a difficult time going beyond those hints in creative idea generation tasks (e.g., Landau \& Lehr, 2004; Smith, Ward \& Schumacher, 1993). When participants in experiments first viewed examples of ideas, they often incorporated the features of the examples in the creative ideas they sketched, a conformity effect. Conformity effects occurred even when participants were asked to think of ideas as different from the examples as possible. These studies show that hints or examples can constrain the creative process. Furthermore, as in the Smith \& Tindell (1997) experiments, this conformity effect cannot be voluntarily avoided; fixating ideas are apparently brought to mind by implicit memory processes.

\subsection{Brainstorming}

Brainstorming refers to creative ideation or idea generation activities done as collaborative groups. The practice of brainstorming, as well as other group creative ideation techniques, has become increasingly popular since the method was originally conceived (Osborn, 1957; Parnes \& Meadow, 1950). Scientific evidence, however, has shown that group brainstorming is less productive than individual brainstorming (Diehl \& Stroebe, 1987, 1991), comparing real groups with nominal groups (i.e., the summed products of the same number of individuals who work individually). A productivity deficit in group brainstorming has been reported ofen; that is, nominal groups generate more non-redundant ideas than do real groups. Kohn \& Smith (2010) showed that such deficits are caused in part by fixation and conformity effects, because group members can become fixated on the ideas they hear from others in their group.

\subsection{Design Fixation}

Studies of design fixation show that the fixation and conformity effects that occur when people solve puzzles or generate creative ideas can be observed when people design new objects or devices to fulfill specified design functions (Jansson \& Smith, 1991). Examples of flawed designs (the flaws were not pointed out or explained to students) that were shown to engineering design students were often incorporated in students' designs when they were asked to design new bicycle carriers, and measuring cups for visually impaired persons. Although students were instructed not to give designs with drinking straws or mouthpieces in a spill-proof coffee cup, many who saw an example of such a design (Fig 1 panel c) produced designs with those explicitly forbidden flaws. Design fixation effects were also observed in professional engineers, as well. Engineers who were shown a highly flawed design for a biomechanical device incorporated the exemplified flaws in their own designs. Some of the example designs used to induce design fixation in Jansson \& Smith's (1991) study are shown in Figure 1.

Exemplified flaws frequently appeared in participants' sketches when they designed ideas for new types of a. bicycle carriers; b. measuring cups for the blind; c. an inexpensive spill-proof coffee cup; d. a biomechanical device for taking samples of material in the intestine. 


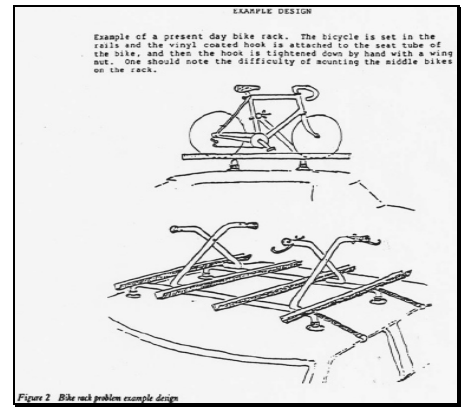

a

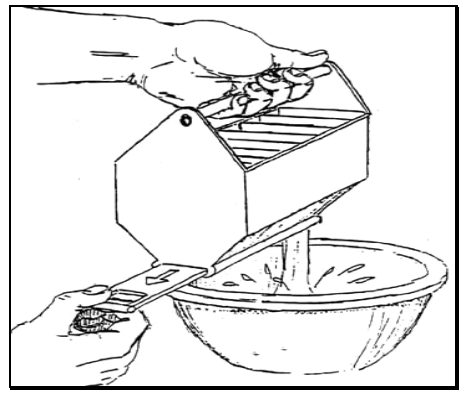

$\mathrm{b}$

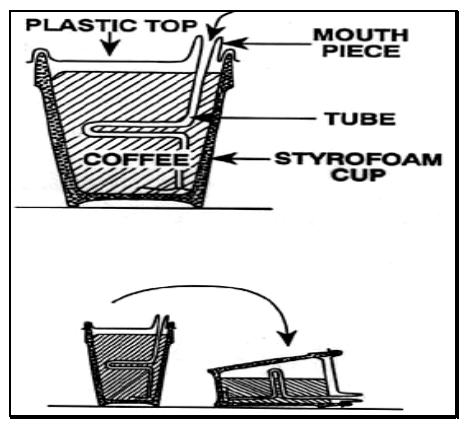

c

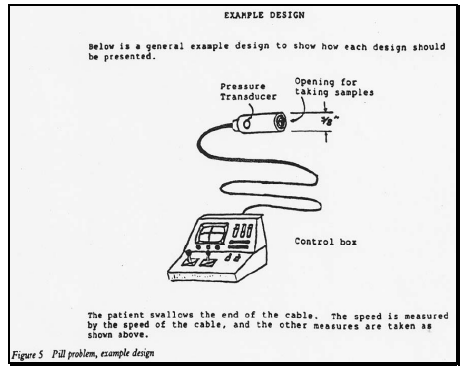

d

Fig. 1. Flawed example designs shown to designers in Jansson \& Smith's (1991) experiments.

\section{Incubation \& Insight}

Insight means a deep understanding of the innermost workings of a problem, which may include critical ideas that can solve difficult problems. When such an understanding springs to mind in a sudden realization, it is referred to as an insight experience, an aha experience, or a eureka moment. Insight experiences are unexpected, yet they are useful for finding ideas critical for solving seemingly intractable problems. Historically significant insights have provided unanticipated solutions to scientific problems, ideas for new products, methods for business practices, and history-changing inventions.

Incubation effects occur when insightful ideas or solutions of problems are realized after difficult problems temporarily are put aside. Anecdotal reports of everyday insight effects are quite common, as when someone puts aside a crossword puzzle when progress is at an impasse, and then instantly realizes the correct answer when they return to the puzzle. A sudden realization that characterizes incubation effects can be an unexpected insight or an unexpected memory. Research has demonstrated incubation effects in memory (Choi \& Smith; 2005; Smith \& Vela, 1991), creative problem solving (Kohn \& Smith, 2009; Smith \& Blankenship, 1989, 1991; Vul \& Pashler, 2007), brainstorming (Kohn \& Smith, 2010), and creative design (Smith, Kohn, \& Shah, 2010).

The two theories best supported by scientific evidence are the forgetting fixation theory (Smith, 1994, 1995) and the opportunistic assimilation theory (Seifert et al., 1995). The opportunistic assimilation theory states that insightful ideas are triggered by stimuli that are serendipitously encountered some time after repeated failures have sensitized one to an unsolved problem. Thus, this theory focuses on hints that point the problem solver towards successful solutions. The forgetting fixation theory states that fixation is a precondition for observing incubation effects; in the absence of fixation, problem solutions are realized in straightforward ways. By putting fixation out of mind, at least temporarily, one can apprehend the problem without the counterproductive influences of inappropriately applied knowledge. This explanation focuses not on pointing towards a solution, but rather on releasing the problem solver from counterproductive work.

\subsection{Forgetting Fixation}

To forget fixation does not require that inappropriately used knowledge is deleted from one's knowledge or memory. Forgetting fixation means to think of the fixated problem without the inappropriate 
information coming to mind. For example, if you are fixated on using a certain approach for solving a physics problem, and that approach is inappropriate for a particular problem, you need not delete that knowledge from memory to solve the problem; you must simply put the fixated approach out of mind when you apprehend the problem in question.

\subsection{Environmental Triggers}

There are many examples of how a clue, accidentally encountered in an unexpected context, triggered an important insight into the solution of a problem. For example, the idea for Velcro came from burrs collected accidentally on a hike through the Alps. As mentioned previously, NASA engineer James Crocker's idea for the Hubble space telescope repair was triggered by a chance encounter he had with an adjustable shower head. An inventor or designer sensitized to problems due to initial failures might stumble onto important clues, as described by the opportunistic assimilation theory (Seifert et al., 1995). In many historic examples, such as those described earlier, it is also the case that unexpected insights usually happen in contexts outside of the workplace. The unusual contexts in which historical insights occur may have caused problem solvers to think of problems in different ways, overcoming the initial fixation. Thus, we have a scientific dilemma: Can fixation be overcome better by shifting contexts, to facilitate thinking about a problem differently, or by exposure to provocative environmental stimuli?

There are fundamental flaws, however, for applying this method as a solution to design fixation. The first problem is that there are so many stimuli in any environment, and of the nearly infinite stimuli one stumbles across every day, which one is the relevant one? The problem is by no means trivial. Second, any one stimulus can be encoded in a very large number of ways. Take, for example, a pair of pliers. It could be encoded as a tool, a grasping tool, a tool for increasing leverage, a piece of metal, a conductive material, a plumb weight, a paperweight, a wedge, a piece of property owned by a carpenter, a human artifact, a substitute for a wrench or a vise-grips, a utensil that could be used to grasp food or dead bugs or a hot pan. Which representation is the one that will help the fixated problem solver? Finally, it is not clear where one should look for a rich source of clues that could trigger insights. A park? A subway station? The internet? What is needed is a place to look for relevant clues that have a good chance of triggering solutions to one's fixated problem.

\subsection{Analogy \& Design}

We propose that a rich source of potentially relevant ideas that could help overcome design fixation is the world of analogy. For example, Crocker's idea for the Hubble space telescope repair was an analogy with an adjustable shower head. Velcro was based on an analogy to burrs. A support for a highway overpass might be based on an analogy to a waiter's hand carrying a heavy tray. Nonetheless, simply looking for any analogy in the world does not narrow the search for a rich source of potentially useful clues for overcoming design fixation. What analogies are most appropriate for a given design problem?

A method for helping engineers identify linguistically remote (cross-domain) analogies, the WordTree Design-by-Analogy Method (Linsey et al., 2008; Linsey, et al., 2009), is based on the cognitive principles of analogical retrieval. Design problems are represented in multiple linguistic representations at various levels of abstraction to maximize the number of appropriate analogues.

\section{Well-Tested Analogies}

Analogies can be based on mechanisms that have been well-tested, and that reliably solve certain problems. Two types of well-tested mechanisms, those found in a patent database, and those found in the domain of biology, are proposed as potential remedies to design fixation. These mechanisms are quite varied and highly imaginative. This speculative proposal is not to adopt these mechanisms by simply plugging them in to one's design, but rather to examine them in a more general abstract manner, the way that analogies can provide useful structure for conceptual design.

One type of database that could be very useful for triggering ideas that might remediate design fixation is a patent database. The patent approval process is clearly one that rigorously tests the efficacy of patented ideas. Such ideas utilize a variety of mechanisms to solve longstanding problems. Accessing patent databases, therefore, provides a rich domain of well-tested ideas that could potentially help the designer overcome design fixation. Linsey et al. (2008) have described the basis of remote analogical transfer from such a database.

Another type of database that could provide a vast source of mechanisms suitable for analogical transfer would be a compendium of life forms, including microorganisms. The long process of evolution, that is, random variation and adaptive selection, has provided a first rate testing ground for the efficacy and adaptability of these life forms. These biological 
mechanisms can provide a rich source of life-based solutions to longstanding environmental problems, such as those related to locomotion, energy transfer, temperature regulation, self-repair, and so on.

Should the designer simply peruse these databases, the way shoppers browse through a mall or readers browse through bookstores or libraries? Perhaps, but we speculate that there may be better ways to find remedies for design fixation, ways that narrow the search for ideas to the most relevant ones that the designer may not have considered. Specifically, the WordTree Method (Linsey et al., 2008; Linsey, et al., 2009) uses abstracted versions of design functions to focus a search for relevant analogues to design problems. Applied to rich sources of adaptive mechanisms, such as patent databases and life form collections, we speculate that designers could have a useful remedy for overcoming design fixation.

\section{References}

Choi, H., \& Smith, S. M. (2005). Incubation and the resolution of Tip-of-the-tongue states. The Journal of General Psychology, 132(4), 365-376.

Chrysikou, E.G., and Weisberg, R.W., (2005). Following the wrong footsteps: fixation effects of pictorial examples in a design problem-solving task. Journal of Experimental Psychology, Learning, Memory and Cognition Vol.31 (5), pp. 1134-1148.

Diehl, M., \& Stroebe,W. (1987). Productivity loss in brainstorming groups: Toward the solution of a riddle. Journal of Personality \& Social Psychology, 497-509.

Diehl, M., \& Stroebe, W. (1991). Productivity loss in ideagenerating groups: Tracking down the blocking effect. Journal of Personality and Social Psychology, 61, 392403.

Finke, RA, Ward, TB, \& Smith, SM (1992). Creative Cognition: Theory, Research, and Applications, Cambridge, MA: MIT Press.

Jansson, D., and Smith, S., 1991, Design fixation. Design Studies Vol.12 (1), pp. 3-11.

Kinoshita, S., \& Towgood, K. (2001). Effects of dividing attention on the memory-block effect. Journal of Experimental Psychology: Learning, Memory, and Cognition, 27, 889_895.

Kohn, N. W., \& Smith, S. M. (2009). Partly versus completely out of your mind: effects of incubation and distraction on resolving fixation. Journal of Creative Behavior, 43(2), 102-118.

Kohn, N. W., \& Smith, S. M. (2010). Collaborative fixation: Effects of others' ideas on brainstorming. Applied Cognitive Psychology.

Landau, J. D., \& Lehr, D. P. (2004). Conformity to experimenter-provided examples: Will people use an unusual feature? Journal of Creative Behavior, 38(3), 180-191.
Landau, J. D., \& Leynes, P. A. (2006). Do explicit memory manipulations affect the memory blocking effect? American Journal of Psychology, 119, 463479.

Leynes, P. A., Rass, O., \& Landau, J. D. (2008). Eliminating the memory blocking effect. Memory, 16(8), 852-872.

Linsey, J., Wood, K., and Markman, A. (2008). Increasing Innovation: Presentation and Evaluation of the WordTree Design-by-Analogy Method. ASME IDETC Design Theory \& Methodology Conference, New York.

Linsey, J., Tseng, I., Fu, K., Cagan, J., and Wood, K., 2009, Reducing and perceiving design fixation: Initial results from a NSF-sponsored workshop. International Conference on Engineering Design, Stanford, California.

Osborn, A. (1957). Applied imagination. New York: Scribner.

Parnes, S. J., \& Meadow, A. (1959). Effects of "brainstorming" instructions on creative problem solving by trained and untrained subjects. Journal of Educational Psychology, 50, 171-176.

Purcell, A. T., and Gero, J. S., (1996). Design and other types of fixation. Design Studies Vol.17 (4), 363-383.

Seifert, C. M., Meyer, D. E., Davidson, N., Patalano, A. L., \& Yaniv, I. (2005). Demystification of cognitive insight: Opportunistic assimilation and the prepared-mind perspective. In R. J. Sternberg \& J. E. Davidson (Eds.) The nature of insight (pp. 65-124). Cambridge, MA: MIT Press.

Smith, S. M. (1994). Getting Into and Out of Mental Ruts: A theory of Fixation, Incubation, and Insight. In The Nature of Insight, (Sternberg, R.J., and Davidson, J., Eds.). MIT Press, Cambridge, MA.

Smith, S. M., (1995). Fixation, incubation, and insight in memory and creative thinking. In The Creative Cognitive Approach, Smith, S. M., Ward, T. B., \& Finke, R. A. (Eds.), pp. 135-156. The MIT Press, Cambridge, MA.

Smith, S. M., and Blankenship, S.E. (1989). Incubation effects. Bulletin of the Psychonomic Society, Vol.27 (4), pp. 311-314.

Smith, S. M., \& Blankenship, S.E. (1991). Incubation and the persistence of fixation in problem solving. American Journal of Psychology, 104, 61-87.

Smith, S. M., Kohn, N. W., and Shah, J. J. (in press). What you see is what you get: Effects of provocative stimuli in creative invention. In Studying design creativity, (Gero, J., Ed.). Springer Press.

Smith, S. M., \& Tindell, D.R. (1997). Memory blocks in word fragment completion caused by involuntary retrieval of orthographically similar primes. Journal of Experimental Psychology: Learning, Memory and Cognition, 23(2), 355-370.

Smith, S. M., \& Vela, E. (1991). Incubated reminiscence effects. Memory \& Cognition, 19 (2), 168-176.

Smith, SM, Ward, TB, \& Finke, RA (1995). The Creative Cognition Approach. Cambridge: MIT Press.

Smith, S. M., Ward, T.B., \& Schumacher, J.S. (1993). Constraining effects of examples in a creative generation task. Memory \& Cognition, 21, 837-845.

Vul, E., \& Pashler, H. (2007). Incubation benefits only after people have been misdirected. Memory \& Cognition, 35(4), 701-710. 